\title{
Combination Treatment of Stereotactic Body Radiation Therapy and Immature Dendritic Cell Vaccination for Augmentation of Local and Systemic Effects
}

\author{
Chul Won Choi, MD' \\ Min Ho Jeong, MD2 \\ You-Soo Park, PhD ${ }^{3}$ \\ Cheol-Hun Son, $\mathrm{PhD}^{3}$ \\ Hong-Rae Lee, $\mathrm{PhD}^{3}$ \\ Eun-Kyoung Koh, MS 3
}

\section{${ }^{1}$ Department of Radiation Oncology,} Dongnam Institute of Radiological \& Medical Sciences, Busan, ${ }^{2}$ Department of Microbiology, Dong-A University College of Medicine, Busan, ${ }^{3}$ Department of Research Center, Dongnam Institute of Radiological $\mathcal{E}$ Medical Sciences, Busan, Korea

\begin{abstract}
Purpose
The purpose of this study was to investigate the efficacy of stereotactic body radiation therapy (SBRT) as a tumor-associated antigen (TAA) presentation method for dendritic cell (DC) sensitization and evaluate its effect in combination with immunotherapy using an intratumoral injection of immature DCs (iDCs).
\end{abstract}

\section{Materials and Methods}

CT-26 colon carcinoma cell was used as a cancer cell line. Annexin V staining and phagocytosis assays were performed to determine the appropriate radiation dose and incubation time to generate TAAs. BALB/c mice were used for in vivo experiments. Cancer cells were injected into the right legs and left flanks to generate primary and metastatic tumors, respectively. The mice were subjected to radiation therapy (RT) alone, intradermal injection of electroporated DCs alone, or RT in combination with iDC intratumoral injection (RT/iDC). Tumor growth measurement and survival rate analysis were performed. Enzyme-linked immunospot and cytotoxicity assays were performed to observe the effect of different treatments on the immune system.

\section{Results}

Annexin V staining and phagocytosis assays showed that 15 Gy radiation dose and 48 hours of incubation was appropriate for subsequent experiments. Maximum DC sensitization and T-cell stimulation was observed with RT as compared to other TAA preparation methods. In vivo assays revealed statistically significant delay in the growth of both primary and metastatic tumors in the RT/iDC group. The overall survival rate was the highest in the RT/iDC group.

\section{Conclusion}

The combination of SBRT and iDC vaccination may enhance treatment effects. Clinical trials and further studies are warranted in the future.
Medical Sciences, Jwadong-gil 40, Jangan-eup,

Gijang-gun, Busan 46033, Korea

Tel: 82-51-720-5066

Fax: 82-51-720-5914

E-mail: drasdf19@gmail.com

Received March 30, 2018

Accepted June 1, 2018

Published Online June 6, 2018

\section{Key words}

Stereotactic body radiotherapy, Immunotherapy, Immature dendritic cells, Tumor antigen

\section{Introduction}

Dendritic cells (DCs) are professional antigen-presenting cells (APCs) and are an essential link between the innate and adaptive immune responses. Although malignant tumor cells may be potentially eliminated via the immune system, they display poor antigenicity. However, DCs perform efficient antigen presentation and initiate the immune response by presenting the captured malignant tumor cells to $\mathrm{T}$ cells in the lymphoid tissue. These functions have deemed DCs an essential target for the induction of tumor-specific immune responses [1-3]. Vaccination strategies using DCs have been developed for the efficient elimination of tumor cells. As the first step in this process, tumor-associated antigens (TAAs) are effectively presented to DCs, leading to their sensitization. TAA presentation may be achieved through the ex vivo-generated antigen-loaded DCs or by inducing DCs to directly phagocytose TAAs in vivo. To improve the effect of DC vaccination strategy, antigen sensitization meth- 
ods are important. Various methods are used to present TAAs to DCs in pre-clinical and clinical trials [4]. However, ex vivo-generated antigen-loaded DCs are commonly used to sensitize DCs in a majority of clinical trials, owing to several disadvantages and limitations associated with the clinical use. Using the same method, at our institution, DCs or electroporated DCs were co-cultured with tumor cell lysates as whole-tumor antigens. This method provides an important advantage over tumor-specific antigen approach by providing broader TAAs without the necessity to identify and characterize antigens $[5,6]$. However, the infeasibility of obtaining the tumor tissue by surgery or biopsy may restrict the application of ex vivo-generated antigen-loaded DCs.

Ionizing radiation was recently reported to be used for the in vivo tumor antigen loading with an intratumoral injection of immature DCs (iDCs). This strategy may overcome the aforementioned limitation of ex vivo-generated antigenloaded DCs and induce effective anticancer activity [4,7]. Radiation therapy (RT) has been considered as a local treatment modality for cancer therapy. However, abscopal effects or side effects at sites other than the treatment area following RT were reported [8-10]. Abscopal effect is caused due to the effect of radiation on the immune system. The direct cytotoxic effect of radiation may involve the release of TAAs, resulting in phagocytosis by APCs and subsequent T-cell stimulation [11]. In addition, the release of damage-associated molecular patterns (DAMPs), such as heat shock proteins (HSPs) or high mobility group box 1 (HMGB-1), from dying tumor cells may provoke immune activation [12,13]. Thus, RT for cancer treatment may not only induce a local effect but also cause an immune-mediated systemic reaction through the augmentation of immune response. In particular, stereotactic body radiation therapy (SBRT) with iDC intratumoral injection is known to cause the aforementioned effect. Here, we investigated the efficacy of RT as a method for TAA presentation to induce DC sensitization and evaluated the effect of RT in combination with immunotherapy using iDC intratumoral injection.

\section{Materials and Methods}

We determined the appropriate radiation dose and incubation time through an in vitro assay. The results of the in vitro assay were then applied to in vivo experiments using BALB/c mice. For in vitro and in vivo experiments, the murine CT-26 colon carcinoma cells were used as the cancer cell line.

\section{In vitro assay}

\section{1) Annexin V staining assay}

Cell death was quantified by flow cytometry using the Annexin V fluorescein isothiocyanate (FITC) apoptosis detection kit, according to the manufacturer's instructions (BD Pharmingen, BD Bioscience, San Jose, CA). Briefly, $1 \times 10^{6}$ CT-26 cells were seeded onto cell culture plates. CT-26 cells were harvested at $0,4,8,12,24,48$, and 72 hours after treatment with irradiation (IR) at 5, 10, 15, and 20 Gy using a linear accelerator (Infinity, ELEKTA, London, UK). Cells were washed with phosphate-buffered saline (PBS), suspended in Annexin V-binding buffer, and treated with an Annexin V-FITC solution and propidium iodide (PI) for 15 minutes at room temperature $\left(18-22^{\circ} \mathrm{C}\right)$. The samples were assessed by flow cytometry on FC500 (Beckman Coulter, Fullerton, CA).

\section{2) Preparation of bone marrow-derived DCs}

Bone marrow cells were isolated from tibias and femurs of mice and red blood cells were lysed following treatment with ammonium chloride potassium lysis buffer (Gibco Invitrogen Corporation, Grand Island, NY) at room temperature for 2 minutes. These cells were washed with PBS and cultured in a complete medium (CM) comprising RPMI-1640 medium (Welgene Corporation, Gyeongsan, Korea) supplemented with $10 \mathrm{ng} / \mathrm{mL}$ recombinant mouse interleukin (IL)-4 (R\&D Systems, Menneapolis, MN) and $20 \mathrm{ng} / \mathrm{mL}$ recombinant mouse granulocyte-macrophage colony-stimulating factor (R\&D Systems) at $1 \times 10^{6}$ cells $/ \mathrm{mL}$. On day $3, \mathrm{CM}$ was added to the culture flasks. On day 6, non-adherent cells were harvested by gentle pitting and observed using FC500.

\section{3) Phagocytosis assay}

We cultured CT-26 cells in culture plates for 24 hours. The cells were subjected to varying doses of IR, and the plates were incubated for $0,24,48$, and 72 hours. Cells of each group were harvested and washed thrice with PBS, followed by staining with 5-(and 6)-carboxyfluorescein diacetate succinimidyl ester (CFSE) at a final concentration of $5 \mu \mathrm{M}$. The labeled cells were washed thrice with PBS. DCs were harvested and stained with anti-mouse CD11c (BD Pharmingen) at $4^{\circ} \mathrm{C}$ for 30 minutes, followed by co-culture with irradiated CT-26 cells in a 96-well plate $(200 \mu \mathrm{L} /$ well $)$ for 2 hours. The cells were analyzed with FC500.

\section{4) Preparation of TAAs}

Irradiation of CT-26 cells was initiated using a linear accelerator. Irradiated CT-26 cells were seeded at a density of 
Tumor injection (CT-26 murine colon carcinoma)

Right thigh $\left(2 \times 10^{6}\right.$ cells $)$

Left flank $\left(1 \times 10^{6}\right.$ cells $)$

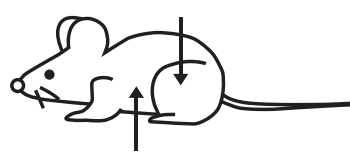

\begin{tabular}{|c|c|c|}
\hline After 7 days & & After 2 days \\
\hline $\begin{array}{c}\text { Right thigh } \\
\text { (tumor: } 50-100 \mathrm{~mm}^{3} \text { ) }\end{array}$ & on right thigh & \\
\hline
\end{tabular}

iDCs i.t injection $\left(2 \times 10^{6}\right.$ cells $)$

$\mathrm{mDC}$ i.d injection $\left(2 \times 10^{6}\right.$ cells $)$

[1st immunization]
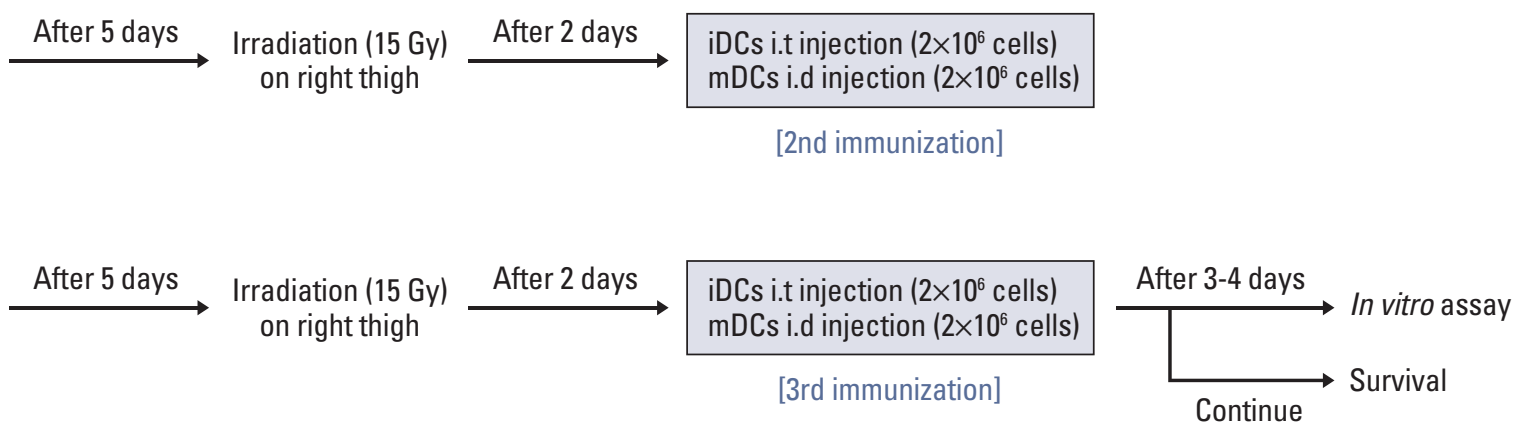

Fig. 1. Schematic diagram of the combination treatment with irradiation and immature dendritic cell injection. Murine CT-26 colon carcinoma cells were inoculated into the right hind legs and left flanks of BALB/c mice. After 7 days of injection, treatment was initiated according to the treatment protocol of each group. The treatment was repeated weekly for three cycles.

$1 \times 10^{6}$ cells in CM. After 3 days, the cells were harvested and centrifuged at $18,400 \times \mathrm{g}$ for 30 minutes to obtain TAAs. A sonicator was used for the induction of tumor cell lysates. CT-26 cells were seeded and sonicated for 10 minutes, followed by their centrifugation at 14,000 rpm for 30 minutes. The protein content of the tumor cell lysates was determined by the Bradford method. The absorbance was measured at a wavelength of $595 \mathrm{~nm}$ with SoftMax Pro 4.3.1 software (Molecular Devices, Sunnyvale, CA).

\section{5) Cytokine release assay}

Mouse IL-10, IL-12, and interferon (IFN)- $\gamma$ enzyme-linked immunosorbent assay kits (CUSABIO, Wuhan, China) were used, according to the manufacturer's instructions. DCs were co-cultured with irradiated CT-26 cells in six-well plates for 5 days. The cell supernatant was harvested in $1.5 \mathrm{~mL}$ tubes, and data were analyzed with Soft Max Pro Software.

\section{6) Cytotoxicity assay}

Splenocytes were isolated from mice and re-stimulated by co-culturing with DCs for 5 days. CT-26 cells were stained using CFSE at a final concentration of $5 \mu \mathrm{M}$. The labeled cells were washed thrice with PBS and re-suspended in CM sup- plemented with $100 \mathrm{U} / \mathrm{mL}$ of recombinant mouse IL-2 (R\&D Systems). Stimulated splenocytes (effector cells) were co-cultured with CT-26 cells (target cells) at an appropriate effector-to-target cell count in a round-bottomed tube (200 $\mu \mathrm{L} /$ tube) for 6 hours at $37^{\circ} \mathrm{C}$ in a humidified atmosphere containing 5\% $\mathrm{CO}_{2}$. Following incubation, the tubes were treated with PI for 5 minutes at room temperature and analyzed with FC500.

\section{In vivo assay}

A metastatic model was prepared using BALB/c mice. Murine CT-26 colon carcinoma cells were injected into the right hind legs and left flanks of mice at $2 \times 10^{6}$ and $1 \times 10^{6}$ cells, respectively. After 7 days from inoculation, tumor volumes were measured using the following formula: tumor volume $\left(\mathrm{mm}^{3}\right)=$ long axis $(\mathrm{mm}) \times$ short axis ${ }^{2}\left(\mathrm{~mm}^{2}\right) / 2$. Mice were divided into four groups, namely, the control, RT alone (RTA), mature DC (mDC), and RT in combination with iDC intratumoral injection (RT/iDC) group. RTA group was treated with $\mathrm{RT}$ alone, while $\mathrm{mDC}$ group received an intratumoral injection of electroporated DCs $\left(2 \times 10^{6}\right.$ cells $)$ co-cultured with tumor cell lysates. Mice in the RT/iDC group were subjected to irradiation followed by an intratumoral injection of iDCs $\left(2 \times 10^{6}\right.$ cells). RT was administered to tumors at right hind 
legs only when RT was indicated and tumors at left flanks received no local treatment to observe the systemic effects. All groups received each treatment thrice at an interval of 7 days. The radiation dose and time duration between irradiation and DC injection were determined from the results of the in vitro assay. The schematic of the experiment schedule is described in Fig. 1.

\section{1) Tumor growth measurement and survival analysis}

Tumor volumes in the right hind legs and left flanks were recorded after tumor cell inoculation at an interval of 2 or 3 days. Each treatment was initiated after the tumor volumes in the right hind legs reached $50-100 \mathrm{~mm}^{3}$. After first treatment, tumor volumes at both locations were continuously measured, and statistical analyses were performed to verify the differences among treatment groups. The number of days for which the mice survived (survival days) was counted from the day of treatment inception and overall survival (OS) rates were calculated using the Kaplan-Meier method.

\section{2) Enzyme-linked immunospot (ELISpot) assay}

Immunospot plates for ELISpot (Merck Millipore, Darmstadt, Germany) were coated overnight at $4^{\circ} \mathrm{C}$ with specific anti-mouse IFN- $\gamma$ antibody dissolved in PBS. The plates were blocked with bovine serum albumin $(10 \mathrm{mg} / \mathrm{mL}$ in PBS) for 1 hour and washed thrice with PBS. Isolated splenocytes (0.5 to $1 \times 10^{6}$ cells/well) from each treatment group and CT-26 murine colon carcinoma cell lysates $(50 \mathrm{mg} / \mathrm{mL})$ were seeded in each well. After incubation at $37^{\circ} \mathrm{C}$ for 24 hours, the plate was washed thrice with PBS and then thrice with PBS-Tween buffer. Cells were treated with biotinylated detection antibodies for 2 hours at room temperature. The plate was washed with PBS-Tween buffer and incubated with streptavidin-horseradish peroxidase $(100 \mu \mathrm{L} /$ well) for 2 hours at room temperature. After washing the plate, a chromogenic substrate and hydrogen peroxide $\left(\mathrm{H}_{2} \mathrm{O}_{2}\right)$ were added to each well to produce spots. Once spots were adequately developed, distilled water was added to terminate the reaction. The plate was air dried overnight and the number of spots was counted, and images were captured using AID ELISpot Reader System (Autoimmun Diagnostika $\mathrm{GmbH}$, Germany).

\section{Ethical statement}

Experiments using animals were approved by the Dongnam Institute of Radiological \& Medical Sciences Institutional Animal Care and Use Committee.

\section{Results}

\section{In vitro assay}

Annexin V staining and phagocytosis assays were performed to determine the appropriate radiation dose and incubation time for the efficient generation of TAAs. In Annexin V staining assay, apoptosis increased in proportion with the increment in the radiation dose and incubation time (Fig. 2A). Although the rate of apoptosis gradually increased with time, the difference in apoptosis rates between two time intervals after 48 hours of incubation was very small. In addition, apoptosis increased with the radiation dose and differences in apoptosis rates were not observed for 15 and 20 Gy after the 48-hour incubation following irradiation. Similar results were observed for the phagocytosis assay (Fig. 2B). Phagocytosis increased with an increment in the radiation dose and incubation time; however, statistically significant differences were not observed beyond the 48-hour incubation duration with 15 Gy radiation dose. Thus, we determined 15 Gy and 48 hours as the appropriate radiation dose and incubation time, respectively, for subsequent experiments.

Cytokine release and cytotoxicity assays were performed to verify the effectiveness of irradiation for DC sensitization and T-cell stimulation. Tumor cell lysates were used as the positive control in these experiments. In the cytokine release assay, co-culture of DCs and irradiated CT-26 cells resulted in a significant increase in the release of IL-12 and IFN- $\gamma$ as compared to other groups but yielded lower levels of IL-10 (Fig. 3). The level of IL-10 released was the highest in the negative control group and lowest for the group subjected to DC and irradiation treatment. IL-12 is the most crucial cytokine that drives the development of naïve T cells into Th1 cells [14]. In contrast, IL-10 is involved in down-regulating DC antigen-presenting function and inducing T-cell tolerance [15]. Therefore, the cytokine release assay confirmed that DC sensitization and T-cell stimulation were maximum following irradiation as compared to other methods. Cytokine assay was performed using isolated splenocytes and co-cultured DCs. The results of the cytotoxicity assay were in line with those of the cytokine release assay. The cytotoxicity against CT-26 cells was significantly higher in the group treated with DCs and irradiation, followed by the positive control group (S1 Fig.).

\section{In vivo assay}

Tumor volumes in the right hind legs and left flanks of mice were measured from the date of tumor cell inoculation at an interval of 2 or 3 days. Each treatment was implemen- 
A
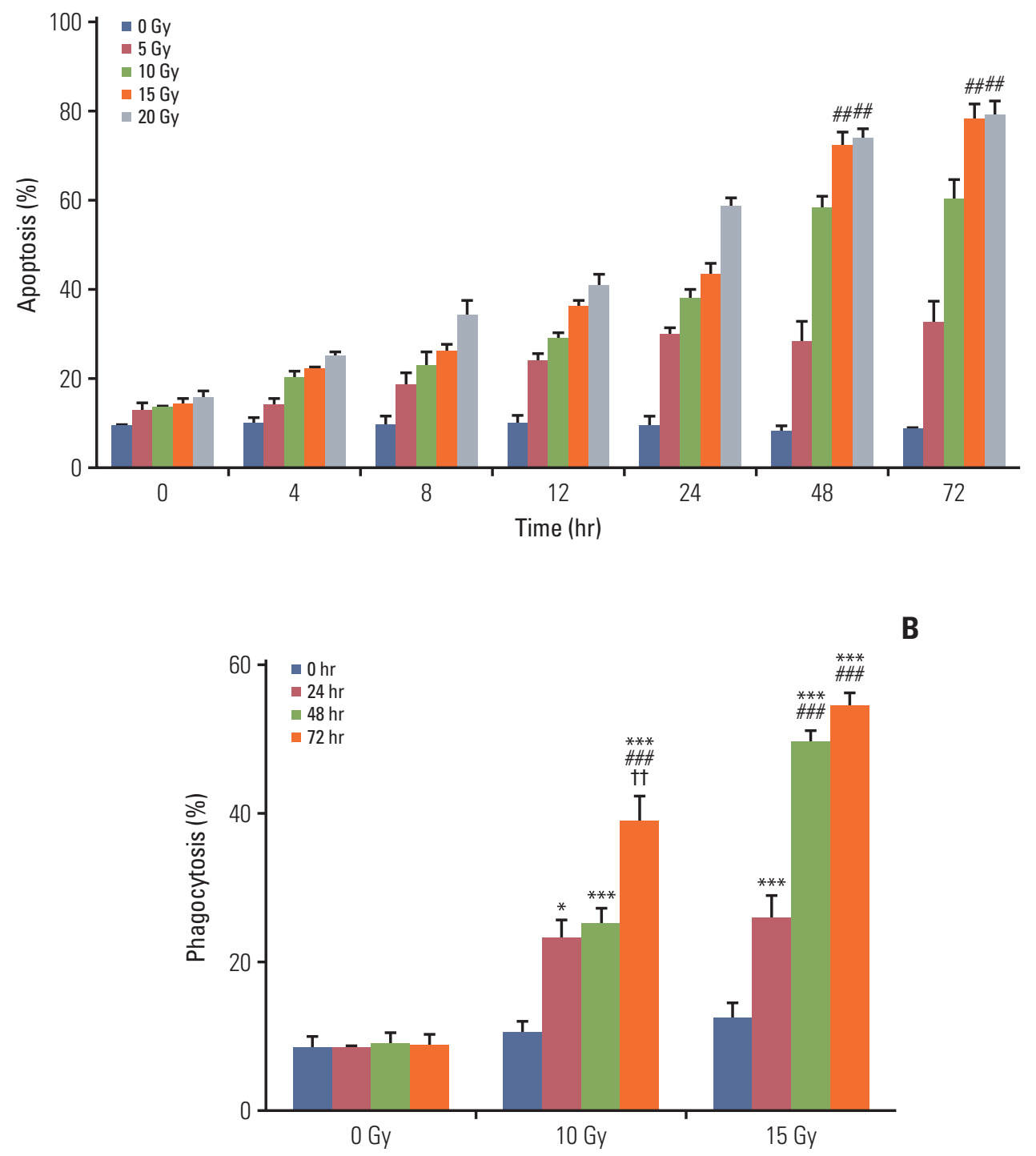

Fig. 2. Annexin V staining assay and phagocytosis assay. (A) Annexin V staining assay. Percentage apoptosis after irradiation increased with an increase in the radiation dose and incubation time. A slight difference was observed between 48 and 72 hours of incubation time. After 48-hour incubation, 15 and $20 \mathrm{~Gy}$ of irradiation had no significant difference in the percentage of apoptosis and exhibited significantly higher effect than 10 Gy of irradiation $(\mathrm{p}<0.01) .10 \mathrm{~Gy}$ vs. 15 or $20 \mathrm{~Gy}\left({ }^{\# \#} \mathrm{p}<0.01\right)$. (B) Phagocytosis assay. A statistically significant increase in the percentage of phagocytosis was observed with an increase in the radiation dose within 48-hour incubation. After 48-hour incubation, 15 and 20 Gy of radiation showed no difference in phagocytosis. 0-Hour incubation vs. other groups ( $\left.{ }^{*} \mathrm{p}<0.05,{ }^{* * *} \mathrm{p}<0.001\right), 24$-hour incubation vs. 48- or 72-hour incubation $\left({ }^{\# \# \#} \mathrm{p}<0.001\right), 48$-hour incubation vs. 72-hour incubation $\left({ }^{++} \mathrm{p}<0.01\right)$.

ted, as previously described. In comparison with the control group, the treatment groups showed statistically significant difference in tumor regression at right hind legs. The groups receiving local RT treatment showed the most remarkable tumor regression; moreover, the $\mathrm{mDC}$ group also showed tumor regression in the absence of local RT treatment (Fig. 4).
Tumor regression was not observed at the left flanks in the RTA, and there was no difference in tumor volumes between this group and the control group. The most definite delay in the growth of tumors at the left flanks was observed in RT/iDC group, while statistically significant growth delay was observed on the $\mathrm{mDC}$ group compared to that in the 

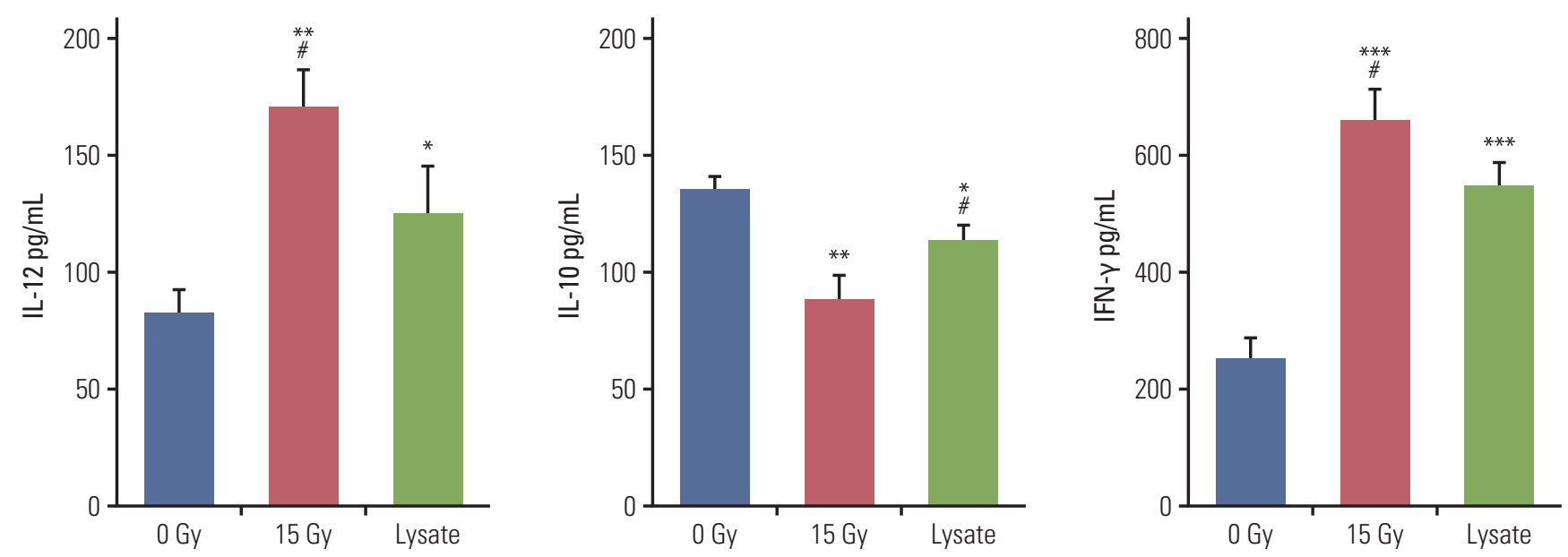

Fig. 3. Cytokine release assay. The assay was performed to verify the effectiveness of irradiation as a method to sensitize dendritic cells to present tumor-associated antigens and stimulate T cells. Tumor cell lysates were used as the positive control. The group irradiated with 15 Gy showed significantly higher interleukin (IL)-12 and interferon $\gamma$ (IFN- $\gamma$ ) levels and lower IL-10 level than other groups. Murine CT-26 colon carcinoma cells alone with or without irradiation did not show detectable levels of the cytokines. 0 Gy vs. other groups ( ${ }^{*} \mathrm{p}<0.05,{ }^{* *} \mathrm{p}<0.01$, $\left.{ }^{* * *} \mathrm{p}<0.001\right), 15$ Gy vs. lysate $\left({ }^{\#} \mathrm{p}<0.05\right)$.

A
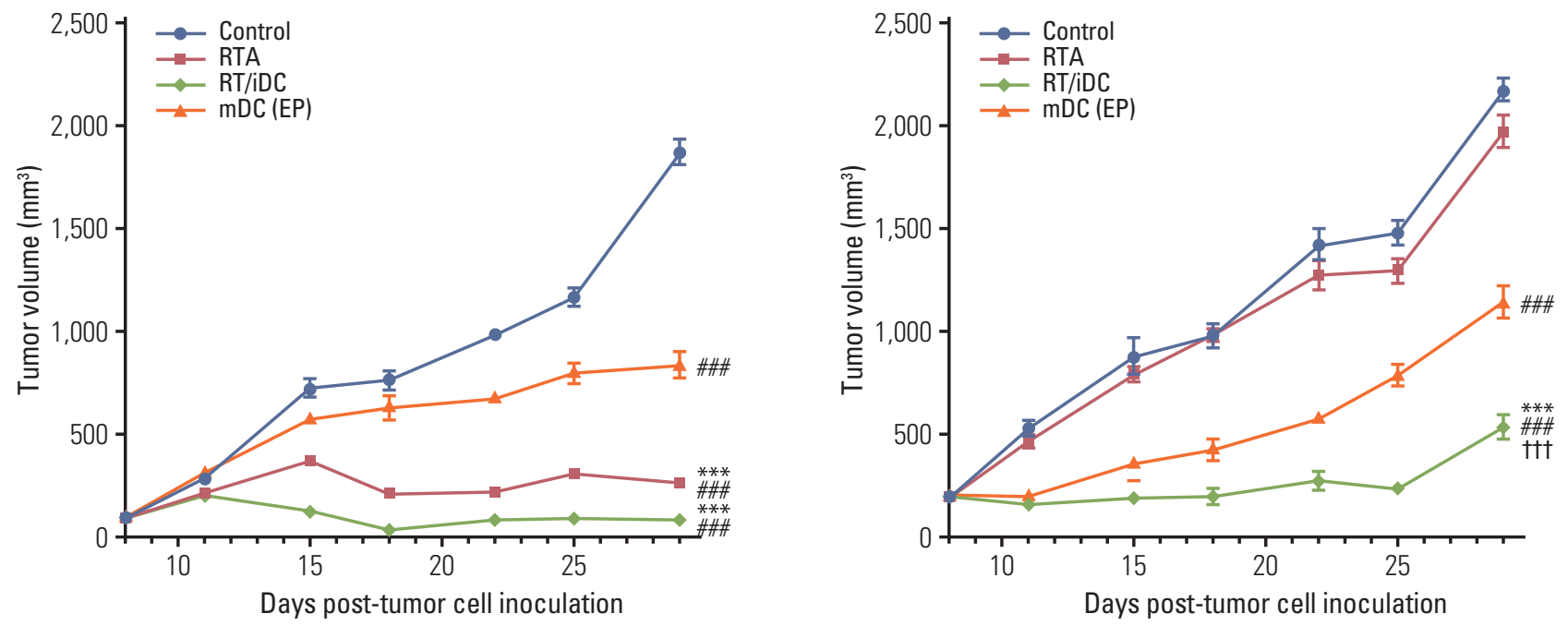

Fig. 4. Volumes of right thigh and left flank tumors. Volumes of the right thigh tumors and the left flank tumors considered as primary tumors and distant metastatic tumors, respectively and both were measured from the date of tumor cell inoculation at an interval of 2 or 3 days. (A) Volumes of the right thigh tumors. Statistically significant difference in the tumor growth delay was observed in all groups compared with the control group. Maximum tumor growth regression was observed in the radiation therapy in combination with immature dendritic cell intratumoral injection (RT/iDC) and radiation therapy alone (RTA) groups; statistically significant difference was not reported between these two groups. (B) Volumes of the left flank tumors. The tumor growth regression was statistically significant in the RT/iDC and mature dendritic cell (mDC) groups. No difference was observed between the tumor volumes in the RTA and control groups. The RT/iDC group showed significant tumor growth delay compared with the mDC group $(\mathrm{p}<0.001)$. Control vs. other groups $\left({ }^{* * *} \mathrm{p}<0.001\right)$, RTA vs. $\mathrm{mDC}$ or RT $/ \mathrm{iDC}\left({ }^{\# \#} \mathrm{p}<0.001\right), \mathrm{mDC}$ vs. RT $/ \mathrm{iDC}\left({ }^{++t} \mathrm{p}<0.001\right)$. 


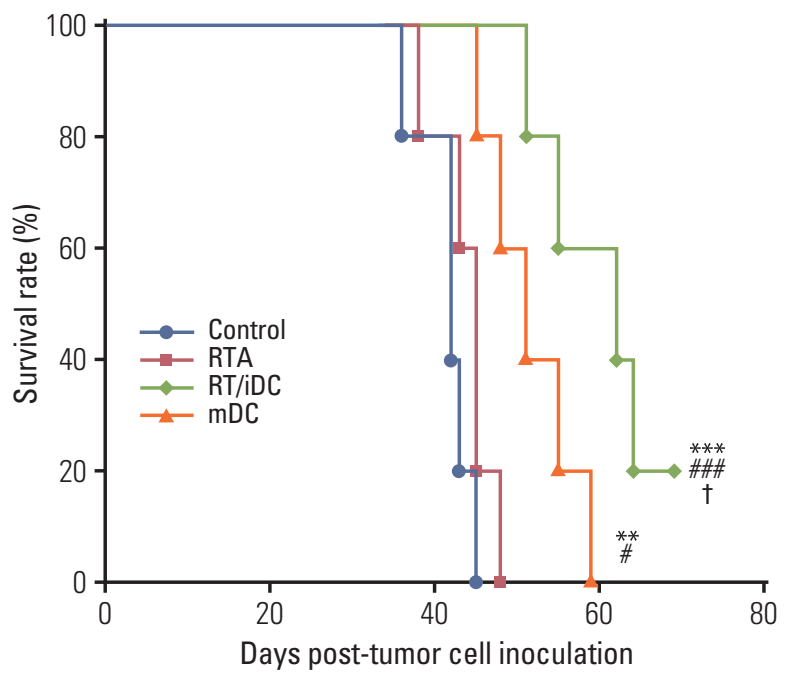

Fig. 5. Kaplan-Meier survival curve. Overall survival rate was prolonged in the radiation therapy in combination with immature dendritic cell intratumoral injection (RT/ iDC) and mature dendritic cell (mDC) groups. There was no survival gain in the radiation therapy alone (RTA) group. Most prolonged overall survival rates were observed in the RT/iDC group. Control vs. other groups $\left({ }^{* *} \mathrm{p}<0.01,{ }^{* * *} \mathrm{p}<0.001\right)$, RTA vs. $\mathrm{mDC}$ or RT $/ \mathrm{iDC}\left({ }^{*} \mathrm{p}<\right.$ $\left.0.05,{ }^{\# \# \#} \mathrm{p}<0.001\right), \mathrm{mDC}$ vs. RT $/ \mathrm{iDC}\left({ }^{\dagger} \mathrm{p}<0.05\right)$.

control group (Fig. 4).

The OS curve was calculated using the Kaplan-Meier method. The results of the survival rate estimation were consistent with those of the left flank tumor volumes. RT/iDC group had the most prolonged OS rate, while $\mathrm{mDC}$ group showed prolonged OS as compared with the control group. However, no survival gain was reported in the RTA group (Fig. 5).

We performed ELISpot assays to evaluate the effect of each treatment on T-cell stimulation. As stimulated T cells secrete IFN- $\gamma$, the analysis of IFN- $\gamma$ secretion using ELISpot may reveal the degree of T-cell stimulation according to treatment schemes. The highest secretion of IFN- $\gamma$ was observed in the $\mathrm{RT} / \mathrm{iDC}$ group, while IFN- $\gamma$ secretion was also increased in the mDC and RTA groups (Fig. 6).

We performed the cytotoxicity assay to examine whether the harvested splenocytes from each treatment group may induce immune responses. The results of the cytotoxicity assay were similar to those of ELISpot assay; maximum cytotoxicity against CT-26 carcinoma cells was observed in $\mathrm{RT} /$ iDC group, followed by mDC and RTA groups (S2 Fig.).

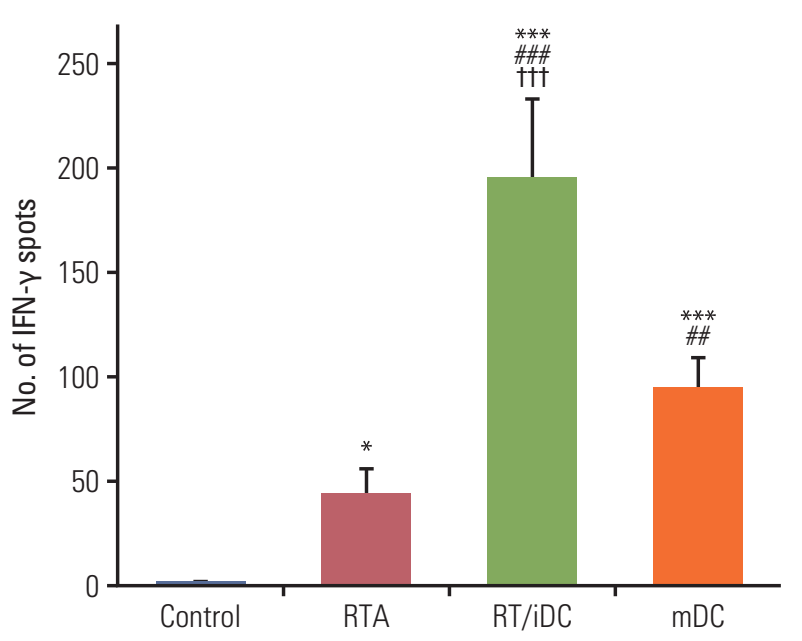

Fig. 6. Tumor-specific interferon $\gamma($ IFN- $\gamma)$-secreting $\mathrm{T}$ cells. IFN- $\gamma$ secretion was assessed using the enzymelinked immunospot assay. As IFN- $\gamma$ secretion increased in the stimulated $\mathrm{T}$ cells, the analysis of IFN- $\gamma$ secretion revealed the degree of T-cell stimulation after each treatment. IFN- $\gamma$ level was the highest in radiation therapy in combination with immature dendritic cell intratumoral injection (RT/iDC) group, followed by the mature dendritic cell (mDC) group. A significant increase in IFN- $\gamma$ level was also observed in the radiation therapy alone (RTA) group $(p<0.05)$. Control vs. other groups $\left({ }^{*} p<0.05\right.$, $\left.{ }^{* * *} \mathrm{p}<0.001\right)$, RTA vs. $\mathrm{mDC}$ or RT $/ \mathrm{iDC}\left({ }^{\sharp \#} \mathrm{p}<0.01,{ }^{\sharp \#} \mathrm{p}<\right.$ 0.001), $\mathrm{mDC}$ vs. RT/iDC $\left({ }^{++t+} \mathrm{p}<0.001\right)$.

\section{Discussion}

Surgery, chemotherapy, and RT have been widely used either in combination or alone for cancer treatment, and several advancements have been reported in these traditional treatment strategies. Nonetheless, the results of cancer treatment still remain unsatisfactory and the associated toxicities are inevitable. There are several challenges to improve the prognosis and reduce these adverse effects. In this direction, DC immunotherapy has been considered as a promising cancer treatment and several studies have been conducted.

The preparation of effective DCs with TAAs is one of the key steps for the successful DC immunotherapy. This could be achieved either by culturing DCs with TAA ex vivo or inducing DCs to phagocytose TAAs in vivo [2]. Irradiation of tumor cells was shown to effectively generate TAAs in vivo and enhance the stimulation of human DCs. Kim et al. [4] reported that $\gamma$-irradiation is superior to other inactivation methods, such as freeze-thawing or staurosporine treatment. DCs sensitized with $\gamma$-irradiated cancer cells exhibit effective 
anticancer activity through the direct augmentation of cytotoxicity as well as the indirect activation of $\mathrm{T}$ cells. Weiss et al. [16] revealed autologous tumor cells as a suitable source of TAAs for vaccination and suggested that therapy-induced alterations at tumor cell surface may expose tumors to the immune system. Furthermore, Chi et al. [17] suggested the advantage of in vivo over ex vivo methods, given the difficulty involved in obtaining sufficient quantities of tumor cells for loading DCs ex vivo. However, there are few comparative studies between in vivo and ex vivo strategies as a TAA presenting method. In addition, there is little research on the combination of RT and iDC intratumoral injection. In the present study, we evaluated the efficacy of RT as a method for in vivo TAA generation and analyzed the effects of treatment comprising RT and immunotherapy using intratumoral iDC injection.

We determined the appropriate radiation dose and incubation time for the generation of TAAs to be 15 Gy and 48 hours, respectively, through in vitro studies. We examined the cytokine release and cytotoxicity to evaluate the effects of DC sensitization and T-cell stimulation by irradiation. Through this study, we confirmed that irradiation of tumor cells could be the most effective TAA presentation method.

In vivo experiments were conducted based on the data from the in vitro experiments. BALB/c mice were used for the generation of metastatic models and growth measurement and survival analysis were performed. In this experiment, right hind leg tumors and left flank tumors represented primary and distant metastatic tumors, respectively. The observation of tumor response at each site demonstrated the local and systemic effects of the treatment.

The group receiving local RT treatment showed the most significant reduction in the tumor size at the right hind leg. In addition, $\mathrm{mDC}$ group also showed a significant decrease in the tumor size without RT. During the examination of the systemic effects of each treatment, we found that RT/iDC group showed the most significant regression of tumors at left flanks as compared with other groups. In addition, significant tumor regression was also observed for $\mathrm{mDC}$ group. Thus, the combination of RT and iDC vaccination exhibited synergistic, not additive, effect through the augmentation of the immune response.

Survival rate was significantly prolonged in both RT/iDC and $\mathrm{mDC}$ groups and RT/iDC group had the highest survival rate. However, there was no difference in the survival rate between RTA and control group. Therefore, survival rate seemed to be determined by not only local tumor response but also overall response including distant tumor response, as survival rates matched with the combined responses of hind leg and flank tumors. In other words, the local effect without systemic effect failed to affect the survival rate or change prognosis in metastatic disease.
We performed in vitro ELISpot and cytotoxicity assays to validate whether the aforementioned results were obtained by T-cell stimulation following TAA and iDC interaction. The results of the ELISpot assay showed that all groups exhibited a statistically significant increase in the number of IFN- $\gamma$ spots, with the highest number reported for the RT/ iDC group. As stimulated T cells secrete IFN- $\gamma$, maximum T-cell stimulation was observed in RT/iDC group [18]. Cytotoxicity assay was performed to observe the induction of the immune response by harvested splenocytes from each group. The result was similar to that of ELISpot assay, as the cytotoxicity against CT-26 carcinoma cells was the highest in RT/iDC group. Therefore, the synergistic effects of the combination treatment with RT and iDC vaccination were induced by the augmentation of the immune response.

Conventional methods using lysates in DC immunotherapy demand invasive procedures, such as surgery or biopsy to obtain tumor cells for the production of tumor antigens. Moreover, it is difficult to obtain an adequate number of tumor cells, and these cells may be lost during the production of mature DCs. In contrast, the combination of RT and iDC vaccination eliminated the need to obtain tumor cells, indicative of the potential clinical application of this method in a wide range of patients.

Recent technical advancements in RT allow the use of SBRT to deliver highly conformal and ablative dose for cancer treatment [19]. SBRT offers a few advantages over conventional fractionation $R T$ in combination with $\mathrm{iDC}$ vaccination. First, the large radiation field with conventional RT provokes immunosuppressive reaction by damaging circulating lymphocytes that are highly sensitive to RT [20,21]. SBRT may significantly reduce RT volume and retain more lymphocytes to facilitate T-cell proliferation. Second, the protracted treatment time for RT using conventional fractionation may increase the exposure of circulating lymphocytes to the radiation beam; SBRT, on the other hand, would preserve these circulating lymphocytes [22]. Third, ablative high dose would cause more apoptosis and necrosis, which would generate tumor antigens to activate the immune system. Moreover, RT may trigger necrosis when used as a single high dose, resulting in the release of DAMPs, such as HSP and HMBG-1, that are widely considered as pro-inflammatory and immune-activating molecules [23-25].

Through in vitro and in vivo experiments, we show that the combination of SBRT and iDC vaccination may increase the immunity of cancer patients, leading to a better therapeutic effect. However, this study has some limitations. The metastatic model was generated using a limited number of tumors, and the treatment protocol constructed was different from that used in clinical settings. Nevertheless, the present study confirmed the possibility of using RT in combination with iDC vaccination. In particular, this strategy may have 
potential advantages for the treatment of patients with oligometastasis or oligorecurrence who are not indicated for adjuvant systemic treatment.

In conclusion, SBRT combined with iDC vaccination may enhance both local and systemic effects of treatment and serve as a promising strategy for cancer treatment in the future. Clinical trials and further studies are warranted to evaluate the application of this treatment strategy.

\section{Electronic Supplementary Material}

Supplementary materials are available at Cancer Research and Treatment website (https://www.e-crt.org).

\section{Conflicts of Interest}

Conflict of interest relevant to this article was not reported.

\section{Acknowledgments}

This work was supported by the Dongnam Institute of Radiological \& Medical Sciences (DIRAMS) grant funded by the Korea government (MSIT) (No.50601-2017 and No. 50595-2018).

\section{References}

1. Datta J, Terhune JH, Lowenfeld L, Cintolo JA, Xu S, Roses RE, et al. Optimizing dendritic cell-based approaches for cancer immunotherapy. Yale J Biol Med. 2014;87:491-518.

2. Palucka K, Banchereau J. Cancer immunotherapy via dendritic cells. Nat Rev Cancer. 2012;12:265-77.

3. Zarnani AH, Torabi-Rahvar M, Bozorgmehr M, Zareie M, Mojtabavi N. Improved efficacy of a dendritic cell-based vaccine against a murine model of colon cancer: the helper protein effect. Cancer Res Treat. 2015;47:518-26.

4. Kim SK, Yun CH, Han SH. Enhanced anti-cancer activity of human dendritic cells sensitized with gamma-irradiationinduced apoptotic colon cancer cells. Cancer Lett. 2013;335: 278-88.

5. Asavaroengchai W, Kotera Y, Mule JJ. Tumor lysate-pulsed dendritic cells can elicit an effective antitumor immune response during early lymphoid recovery. Proc Natl Acad Sci U S A. 2002;99:931-6.

6. Fields RC, Shimizu K, Mule JJ. Murine dendritic cells pulsed with whole tumor lysates mediate potent antitumor immune responses in vitro and in vivo. Proc Natl Acad Sci U S A. 1998;95:9482-7.

7. Son CH, Bae JH, Shin DY, Lee HR, Yang K, Park YS. Antitumor effect of dendritic cell loaded ex vivo and in vivo with tumor-associated antigens in lung cancer model. Immunol Invest. 2014;43:447-62.

8. Demaria S, Ng B, Devitt ML, Babb JS, Kawashima N, Liebes $\mathrm{L}$, et al. Ionizing radiation inhibition of distant untreated tumors (abscopal effect) is immune mediated. Int J Radiat Oncol Biol Phys. 2004;58:862-70.

9. Koo T, Kim IA. Radiotherapy and immune checkpoint blockades: a snapshot in 2016. Radiat Oncol J. 2016;34:250-9.

10. Siva S, MacManus MP, Martin RF, Martin OA. Abscopal effects of radiation therapy: a clinical review for the radiobiologist. Cancer Lett. 2015;356:82-90.

11. Demaria S, Formenti SC. Sensors of ionizing radiation effects on the immunological microenvironment of cancer. Int J Radiat Biol. 2007;83:819-25.

12. Deloch L, Derer A, Hartmann J, Frey B, Fietkau R, Gaipl US. Modern radiotherapy concepts and the impact of radiation on immune activation. Front Oncol. 2016;6:141.

13. Derer A, Deloch L, Rubner Y, Fietkau R, Frey B, Gaipl US. Radio-immunotherapy-induced immunogenic cancer cells as basis for induction of systemic anti-tumor immune responses: pre-clinical evidence and ongoing clinical applications. Front Immunol. 2015;6:505.

14. O'Garra A. Cytokines induce the development of functionally heterogeneous T helper cell subsets. Immunity. 1998;8:275-83.

15. Igietseme JU, Ananaba GA, Bolier J, Bowers S, Moore T, Belay $\mathrm{T}$, et al. Suppression of endogenous IL-10 gene expression in dendritic cells enhances antigen presentation for specific Th1 induction: potential for cellular vaccine development. J Immunol. 2000;164:4212-9.

16. Weiss EM, Frey B, Rodel F, Herrmann M, Schlucker E, Voll $\mathrm{RE}$, et al. Ex vivo- and in vivo-induced dead tumor cells as modulators of antitumor responses. Ann N Y Acad Sci. 2010; 1209:109-17.

17. Chi KH, Liu SJ, Li CP, Kuo HP, Wang YS, Chao Y, et al. Combination of conformal radiotherapy and intratumoral injection of adoptive dendritic cell immunotherapy in refractory hepatoma. J Immunother. 2005;28:129-35.

18. Slota M, Lim JB, Dang Y, Disis ML. ELISpot for measuring human immune responses to vaccines. Expert Rev Vaccines. 2011;10:299-306.

19. Benedict SH, Yenice KM, Followill D, Galvin JM, Hinson W, Kavanagh B, et al. Stereotactic body radiation therapy: the report of AAPM Task Group 101. Med Phys. 2010;37:4078-101.

20. Kang J, Demaria S, Formenti S. Current clinical trials testing the combination of immunotherapy with radiotherapy. J Immunother Cancer. 2016;4:51.

21. Sharabi AB, Tran PT, Lim M, Drake CG, Deweese TL. Stereo- 
tactic radiation therapy combined with immunotherapy: augmenting the role of radiation in local and systemic treatment. Oncology (Williston Park). 2015;29:331-40.

22. Yovino S, Kleinberg L, Grossman SA, Narayanan M, Ford E. The etiology of treatment-related lymphopenia in patients with malignant gliomas: modeling radiation dose to circulating lymphocytes explains clinical observations and suggests methods of modifying the impact of radiation on immune cells. Cancer Invest. 2013;31:140-4.
23. Krysko O, Love Aaes T, Bachert C, Vandenabeele P, Krysko DV. Many faces of DAMPs in cancer therapy. Cell Death Dis. 2013;4:e631.

24. Proskuryakov SY, Konoplyannikov AG, Gabai VL. Necrosis: a specific form of programmed cell death? Exp Cell Res. 2003;283:1-16.

25. Vandenabeele P, Galluzzi L, Vanden Berghe T, Kroemer G. Molecular mechanisms of necroptosis: an ordered cellular explosion. Nat Rev Mol Cell Biol. 2010;11:700-14. 